\title{
Parallel Sparse Polynomial Interpolation over Finite Fields
}

\author{
Seyed Mohammad Mahdi Javadi \\ School of Computing Science \\ Simon Fraser University \\ Burnaby, B.C. Canada. \\ sjavadi@cecm.sfu.ca.
}

\author{
Michael Monagan \\ Department of Mathematics \\ Simon Fraser University \\ Burnaby, B.C. Canada. \\ mmonagan@cecm.sfu.ca.
}

\begin{abstract}
We present a probabilistic algorithm to interpolate a sparse multivariate polynomial over a finite field, represented with a black box. Our algorithm modifies the algorithm of BenOr and Tiwari from 1988 for interpolating polynomials over rings with characteristic zero to characteristic $p$ by doing additional probes.

To interpolate a polynomial in $n$ variables with $t$ non-zero terms, Zippel's (1990) algorithm interpolates one variable at a time using $O(n d t)$ probes to the black box where $d$ bounds the degree of the polynomial. Our new algorithm does $O(n t)$ probes. It interpolates each variable independently using $O(t)$ probes which allows us to parallelize the main loop giving an advantage over Zippel's algorithm.

We have implemented both Zippel's algorithm and the new algorithm in $\mathrm{C}$ and we have done a parallel implementation of our algorithm using Cilk [2]. In the paper we provide benchmarks comparing the number of probes our algorithm does with both Zippel's algorithm and Kaltofen and Lee's hybrid of the Zippel and Ben-Or/Tiwari algorithms.
\end{abstract}

Categories and Subject Descriptors:

I.1.2 [Symbolic and Algebraic Manipulation]: Algorithms

General Terms: Algorithms, Theory.

Keywords: sparse polynomial interpolation, parallel interpolation algorithms, Ben-Or Tiwari.

\section{INTRODUCTION}

Let $p$ be a prime and let $f \in \mathbb{Z}_{p}\left[x_{1}, \ldots, x_{n}\right]$ be a multivariate polynomial with $t>0$ non-zero terms which is represented with a black box $\mathbb{Z}_{p}^{n} \rightarrow \mathbb{Z}_{p}$. On input $\left(\alpha_{1}, \ldots, \alpha_{n}\right) \in$ $\mathbb{Z}_{p}^{n}$, the black box evaluates and outputs $f\left(x_{1}=\alpha_{1}, \ldots, x_{n}=\right.$ $\alpha_{n}$ ). Given also a degree bound $d$ on the degree of $f$, our goal is to interpolate the polynomial $f$ with minimum number of evaluations (probes to the black box).

\section{*This work was supported by NSERC of Canada and the MITACS NCE of Canada}

Permission to make digital or hard copies of all or part of this work for personal or classroom use is granted without fee provided that copies are not made or distributed for profit or commercial advantage and that copies bear this notice and the full citation on the first page. To copy otherwise, to republish, to post on servers or to redistribute to lists, requires prior specific permission and/or a fee.

PASCO 2010, 21-23 July 2010, Grenoble, France.

Copyright 2010 ACM 978-1-4503-0067-4/10/0007 ...\$10.00.
Sparse interpolation is a key part of many algorithms in computer algebra such as polynomial GCD computation $[17$, $7,13]$ over $\mathbb{Z}$. Here one computes the GCD modulo $p$ where $p$ is chosen to be a machine size prime. We are interested in algorithms whose computational complexity is polynomial in $t, n, d$ and $\log p$. In 1979 Richard Zippel presented the first such algorithm. Zippel's algorithm is probabilistic. It relies heavily on the assumption that if a polynomial is zero at a random evaluation point, then it is the zero polynomial with high probability. Zippel's algorithm interpolates $f$ one variable at a time, sequentially. It makes $O(n d t)$ probes to the black box. In 1990, Zippel in [18] improved his 1979 algorithm to use evaluation points of the form $\left(\alpha_{1}^{i}, \ldots, \alpha_{k}^{i}\right) \in$ $\mathbb{Z}_{p}^{k}$ so that the linear systems to be solved become transposed Vandermonde systems which can be solved in $O\left(t^{2}\right)$ time instead of $O\left(t^{3}\right)-$ see [8].

In 1988, Ben-Or and Tiwari [1] presented a deterministic algorithm for interpolating a multivariate polynomial with integer, rational, real or complex coefficients. Given a bound $T$ on the number of terms $t$ of the polynomial $f$, the algorithm evaluates the black box at powers of the first $n$ primes; it evaluates at the points $\left(2^{i}, 3^{i}, 5^{i}, \ldots, p_{n}^{i}\right)$ for $0 \leq i<2 T$. If $M_{j}\left(x_{1}, \ldots, x_{n}\right)$ are the monomials of the $t$ non-zero terms of $f$, it then uses Berlekamp/Massey algorithm [12] from coding theory to find the monomial evaluations $M_{j}\left(2,3,5, \ldots, p_{n}\right)$ for $1 \leq j \leq t$ and then determines the degree of each monomial $M_{j}$ in $x_{k}$ by trial division of $M_{j}\left(2,3,5, \ldots, p_{n}\right)$ by $p_{k}$. This algorithm is not variable by variable. Instead, it interpolates the polynomial $f$ with $2 T$ probes to the black box which can all be computed in parallel. The major disadvantage of the Ben-Or/Tiwari algorithm is that the evaluation points are large $(O(T \log n)$ bits long - see [1]) and computations over $\mathbb{Q}$ encounter an expression swell which makes the algorithm very slow. This problem was addressed by Kaltofen et al. in [9] by running the algorithm modulo a power of a prime of sufficiently large size; the modulus must be greater than $\max _{j} M_{j}\left(2,3,5, \ldots, p_{n}\right)$.

In [6], Huang and Rao describe how to make the BenOr/Tiwari approach work over finite fields $\operatorname{GF}(q)$ with at least $4 t(t-2) d^{2}+1$ elements. Their idea is to replace the primes $2,3,5, \ldots, p_{n}$ in Ben-Or/Tiwari by linear (hence irreducible) polynomials in $\operatorname{GF}(q)[y]$. Their algorithm is Las Vegas and does $O\left(d t^{2}\right)$ probes. Although the authors discuss how to parallelize the algorithm, the factor of $t^{2}$ may limit this approach.

In 2000, Kaltofen et al. in $[10,11]$ design a hybrid algorithm, a hybrid of the Zippel and Ben-Or Tiwari algorithms, which they call a "racing algorithm". To reduce the number 
of probes when interpolating the next variable in Zippel's algorithm, their algorithm runs a Newton interpolation and a univariate Ben-Or/Tiwari simultaneously, stopping when the first succeeds. However, this further sequentializes the algorithm. In Section 5, we compare the number of probes made by this algorithm to our new algorithm.

In 2009, Giesbrecht, Labahn and Lee in [4] present two new algorithms for sparse interpolation for polynomials with floating point coefficients. The first is a modification of the Ben-Or/Tiwari algorithm that uses $O(t)$ probes. In principle, this algorithm can be made to work over finite fields $G F(q)$ for applications where one can choose $q$. One needs $q-1$ to have $n$ distinct prime factors all $>d$. One would also need $q-1$ to have no large prime factors so that the discrete logarithms needed could be done efficiently using the Pohlig-Hellman algorithm [14]. We have not explored the feasibility of this approach.

Our approach for sparse interpolation over $\mathbb{Z}_{p}$ is to use evaluation points of the form $\left(\alpha_{1}^{i}, \ldots, \alpha_{n}^{i}\right) \in \mathbb{Z}_{p}^{n}$ and modify the Ben-Or/Tiwari algorithm to do extra probes to determine the degrees of the variables in each monomial in $f$. We do a factor of at most $2 n$ more evaluations in order to recover the monomials from their images. A motivation for our new algorithm is to use the Ben-Or/Tiwari approach in modular algorithms (e.g. GCD computations in characteristic 0 - see [7]) where the prime $p$ is chosen to be a machine prime so that arithmetic in $\mathbb{Z}_{p}$ is efficient.

Our paper is organized as follows. In Section 2 we present an example showing the main flow and the key features of our algorithm. We then identify possible problems that can occur and how the new algorithm deals with them in Section 3. In Section 4 we present our new algorithm and analyze its sequential time complexity. Finally, in Section 5 we compare the $\mathrm{C}$ implementations of our algorithm and Zippel's algorithm with the racing algorithm of Kaltofen and Lee [11] on various sets of polynomials.

\section{THE IDEA AND AN EXAMPLE}

Let $f=\sum_{i=1}^{t} a_{i} M_{i} \in \mathbb{Z}_{p}\left[x_{1}, \ldots, x_{n}\right]$ be the polynomial represented with the black box with $a_{i} \in \mathbb{Z}_{p} \backslash\{0\}$. Here $t$ is the number of non-zero terms in $f . M_{i}=x_{1}^{e_{i 1}} \times x_{2}^{e_{i 2}} \times \cdots \times$ $x_{n}^{e_{i n}}$ is the $i$ th monomial in $f$ where $M_{i} \neq M_{j}$ for $i \neq j$. Let $d \geq \operatorname{deg} f$ be a bound on the degree of $f$ so that $e_{i j} \leq d$ for all $1 \leq i, j \leq n$.

We demonstrate our algorithm on the following example. Here we use $x, y$ and $z$ for variables instead of $x_{1}, x_{2}$ and $x_{3}$.

Example 1 Let $f=91 y z^{2}+94 x^{2} y z+61 x^{2} y^{2} z+42 z^{5}+1$ and $p=101$. Given the number of terms $t=5$, the number of variables $n=3$, a degree bound $d=5$ and the black box that computes $f$, we want to find $f$.

The first step is to pick $n=3$ generators $\alpha_{1}, \alpha_{2}, \alpha_{3}$ of $\mathbb{Z}_{p}^{*}$. We evaluate the black box at the points $\beta_{0}, \ldots, \beta_{2 t-1}$ where $\beta_{i}=\left(\alpha_{1}^{i}, \alpha_{2}^{i}, \ldots, \alpha_{n}^{i}\right)$. Thus we make $2 t$ probes to the black box. The reason to use generators instead of random values from $\mathbb{Z}_{p}$ is that it decreases the probability of two distinct monomials having the same evaluation. For our example, let the generators be $\alpha_{1}=66, \alpha_{2}=12$ and $\alpha_{3}=3$ and let $v_{i}$ be the output of the black box on input $\beta_{i}$ and let $V=$ $\left(v_{0}, \ldots, v_{2 t-1}\right)$. In this example we obtain

$$
V=(87,78,65,41,49,38,87,29,23,86) .
$$

Now we use the Berlekamp/Massey algorithm [12] (See [10] for a more accessible reference). The input to this algorithm is a sequence of elements $b_{0}, b_{1}, \ldots, b_{2 t-1}, \ldots$ where $b_{i} \in \mathbb{Z}_{p}$. The algorithm computes a linear generator for the sequence, i.e. the univariate polynomial $\Lambda(z)=z^{t}-\lambda_{t-1} z^{t-1}-\cdots-\lambda_{0}$ such that

$$
b_{t+i}=\lambda_{t-1} b_{t+i-1}+\lambda_{t-2} b_{t+i-2}+\cdots+\lambda_{0} b_{i}
$$

for all $i \geq 0$. In our example the input is $V=\left(v_{0}, \ldots, v_{2 t-1}\right)$ and the output is

$$
\Lambda_{1}(z)=z^{5}+28 z^{4}+62 z^{3}+54 z^{2}+11 z+46 .
$$

The next step is to find the roots of $\Lambda_{1}(z)$. We know (see [1]) that this polynomial is the product of exactly $t=5$ linear factors. The roots are $r_{1}=1, r_{2}=7, r_{3}=41, r_{4}=61$ and $r_{5}=64$. Ben-Or and Tiwari prove that for each $1 \leq i \leq t$, there exists $1 \leq j \leq t$ such that

$$
m_{i}=M_{i}\left(\alpha_{1}, \ldots, \alpha_{n}\right) \equiv r_{j} \bmod p .
$$

The main step now is to determine the degrees of each monomial in $f$ in each variable. Consider the first variable $x$. Let $\alpha_{n+1}$ be a new random generator of $\mathbb{Z}_{p}^{*}$. In this example we choose $\alpha_{4}=34$. This time we choose the evaluation points $\beta_{0}^{\prime}, \ldots, \beta_{2 t-1}^{\prime}$ where $\beta_{i}^{\prime}=\left(\alpha_{n+1}^{i}, \alpha_{2}^{i}, \ldots, \alpha_{n}^{i}\right)$. Note that this time we are evaluating the first variable at powers of $\alpha_{n+1}$ instead of $\alpha_{1}$. We evaluate the black box at these points and apply the Berlekamp/Massey algorithm on the sequence of the outputs to compute the linear generator for the new sequence

$$
\Lambda_{2}=z^{5}+45 z^{3}+54 z^{2}+60 z+42 .
$$

Let $\bar{r}_{1}, \ldots, \bar{r}_{5}$ be distinct roots of $\Lambda_{2}$.

We know that $M_{i}\left(\alpha_{n+1}, \alpha_{2}, \ldots, \alpha_{n}\right)$ is a root of $\Lambda_{2}$ for $1 \leq$ $i \leq n$. On the other hand we have

$$
\frac{M_{i}\left(\alpha_{n+1}, \alpha_{2}, \ldots, \alpha_{n}\right)}{M_{i}\left(\alpha_{1}, \alpha_{2}, \ldots, \alpha_{n}\right)}=\left(\frac{\alpha_{n+1}}{\alpha_{1}}\right)^{e_{i 1}} .
$$

Let $r_{j}=M_{i}\left(\alpha_{1}, \alpha_{2}, \ldots, \alpha_{n}\right)$ and $\bar{r}_{k}=M_{i}\left(\alpha_{n+1}, \alpha_{2}, \ldots, \alpha_{n}\right)$. From Equation 1 we have

$$
\bar{r}_{k}=r_{j} \times\left(\frac{\alpha_{n+1}}{\alpha_{1}}\right)^{e_{i 1}},
$$

i.e. for every root $r_{j}$ of $\Lambda_{1}, r_{j} \times\left(\frac{\alpha_{n+1}}{\alpha_{1}}\right)^{e_{i 1}}$ is a root of $\Lambda_{2}$ for some $e_{i 1}$ which is the degree of some monomial in $f$ with respect to $x$. This gives us a way to compute the degree of each monomial $M_{i}$ in the variable $x$. In this example we have $\frac{\alpha_{n+1}}{\alpha_{1}}=25$. We start with the first root of $\Lambda_{1}$ and check if $r_{1} \times\left(\frac{\alpha_{n+1}}{\alpha_{1}}\right)^{i}$ is a root of $\Lambda_{2}$ for $0 \leq i \leq d$. For $r_{1}=1$ we have that $r_{1} \times\left(\frac{\alpha_{n+1}}{\alpha_{1}}\right)^{0}$ is a root of $\Lambda_{2}$ and for $0<i \leq d$, $r_{1} \times\left(\frac{\alpha_{n+1}}{\alpha_{1}}\right)^{i}$ is not a root of $\Lambda_{2}$, hence we conclude that the degree of the first monomial of $f$ in $x$ is 0 . We continue this to find the degrees of all the monomials in $f$ in the variable $x$. We obtain

$$
e_{11}=0, e_{21}=0, e_{31}=0, e_{41}=2, e_{51}=2 .
$$

We proceed to the next variable $y$. This time we evaluate the black box at $\beta_{0}^{\prime \prime}, \ldots, \beta_{2 t-1}^{\prime \prime}$ where $\beta_{i}^{\prime \prime}=\left(\alpha_{1}^{i}, \alpha_{n+1}^{i}, \alpha_{3}^{i}, \ldots, \alpha_{n}^{i}\right)$ and apply the Berlekamp/Massey algorithm on the sequence of the outputs to compute

$$
\Lambda_{3}=z^{5}+5 z^{4}+27 z^{3}+36 z^{2}+93 z+40
$$


the linear generator for the new sequence. Let $\tilde{r}_{1}, \ldots, \tilde{r}_{5}$ be distinct roots of $\Lambda_{3}$. Again using the same approach as above, we find that the degrees of the monomials in the second variable $y$ to be

$$
e_{12}=0, e_{22}=1, e_{32}=0, e_{42}=2, e_{52}=1 \text {. }
$$

Finally we proceed to the last variable $z$. This time we evaluate $z$ at powers of $\alpha_{n+1}$ instead of $\alpha_{3}$ and compute the following linear generator for the sequence of outputs obtained by probing the black box

$$
\Lambda_{4}=z^{5}+27 z^{4}+99 z^{3}+18 z^{2}+16 z+41 .
$$

We compute the degrees with the same technique and obtain

$$
e_{13}=0, e_{23}=2, e_{33}=5, e_{43}=1, e_{53}=1 .
$$

The reader may observe that determining the degrees of the monomials $M_{i}$ in each variable represent $n$ independent tasks which can therefore be done in parallel. At this point we have computed all the monomials. Recall that $M_{i}=x_{1}^{e_{i 1}} \times$ $x_{2}^{e_{i 2}} \times \cdots \times x_{n}^{e_{i n}}$ hence we have

$M_{1}=1, M_{2}=y z^{2}, M_{3}=z^{5}, M_{4}=x^{2} y^{2} z$ and $M_{5}=x^{2} y z$.

Now we need to compute the coefficients. We do this by solving one linear system of equations. We computed the roots of $\Lambda_{1}$ and we have computed the monomials such that $M_{i}\left(\alpha_{1}, \ldots, \alpha_{n}\right)=r_{i}$. Recall that $v_{i}$ is the output of the black box on the input $\beta_{i}=\left(\alpha_{1}^{i}, \ldots, \alpha_{n}^{i}\right)$ hence we have

$$
v_{i}=a_{1} r_{1}^{i}+a_{2} r_{2}^{i}+\cdots+a_{t} r_{t}^{i}
$$

for $0 \leq i \leq 2 t-1$. Note that the system of equations obtained from the above set of equations is a Vandermonde system which can be solved in $O\left(t^{2}\right)$ time and $O(t)$ space (See [18]). After solving we obtain

$$
a_{1}=1, a_{2}=91, a_{3}=42, a_{4}=61 \text { and } a_{5}=94
$$

and hence $f=1+91 y z^{2}+42 z^{5}+61 x^{2} y^{2} z+94 x^{2} y z$ is interpolated and we are done.

\section{PROBLEMS}

The evaluation points $\alpha_{1}, \ldots, \alpha_{n}, \alpha_{n+1}$ must satisfy certain conditions for our new algorithm to work properly. Here we identify all problems.

\subsection{Distinct Monomials}

The first condition is that for $i \neq j$

$$
M_{i}\left(\alpha_{1}, \ldots, \alpha_{n}\right) \neq M_{j}\left(\alpha_{1}, \ldots, \alpha_{n}\right) \text { in } \mathbb{Z}_{p}
$$

so that $\operatorname{deg}\left(\Lambda_{1}(z)\right)=t$. Also, at the $k$ 'th step of the algorithm, when computing the degrees of the monomials in $x_{k}$, we must have

$$
\forall 1 \leq i \neq j \leq t, \quad m_{i, k} \neq m_{j, k} \quad \text { in } \mathbb{Z}_{p}
$$

where $m_{i, k}=M_{i}\left(\alpha_{1}, \ldots, \alpha_{k-1}, \alpha_{n+1}, \alpha_{k+1}, \ldots, \alpha_{n}\right)$ so that $\operatorname{deg}\left(\Lambda_{k+1}(z)\right)=t$. To reduce the probability of monomial evaluations colliding, we pick $\alpha_{i}$ to have order $>d$. The easiest way to do this is to use generators of $\mathbb{Z}_{p}^{*}$. There are $\phi(p-1)$ generators where $\phi$ is Euler's totient function. We now give an upper bound on the probability that no monomial evaluations collide when we use generators for evaluations.
Theorem 1 Let $\alpha_{1}, \ldots, \alpha_{n}$ be generators from $\mathbb{Z}_{p}$ chosen at random and let $m_{i}=M_{i}\left(\alpha_{1}, \ldots, \alpha_{n}\right)$. Then the probability that two or more monomials evaluate to the same value (we get a collision) is

$$
\leq\left(\begin{array}{l}
t \\
2
\end{array}\right) \frac{d}{\phi(p-1)}<\frac{d t^{2}}{2 \phi(p-1)} .
$$

Proof. Consider the polynomial

$$
A=\prod_{1 \leq i<j \leq t}\left(M_{i}\left(x_{1}, \ldots, x_{n}\right)-M_{j}\left(x_{1}, \ldots, x_{n}\right)\right) .
$$

Observe that $A\left(\alpha_{1}, \ldots, \alpha_{n}\right)=0$ iff two monomial evaluations collide. Recall that the Schwartz-Zippel lemma ([16, 17]) says that if $r_{1}, \ldots, r_{n}$ are chosen at random from any subset $S$ of a field $K$ and $F \in K\left[x_{1}, \ldots, x_{n}\right]$ is non-zero then

$$
\operatorname{Prob}\left(F\left(r_{1}, \ldots, r_{n}\right)=0\right) \leq \frac{\operatorname{deg} f}{|S|} .
$$

Our result follows from noting that $d \geq \operatorname{deg} f$ and thus $\operatorname{deg} A \leq\left(\begin{array}{l}t \\ 2\end{array}\right) d$ and $|S|=\phi(p-1)$, the number of primitive elements in $\mathbb{Z}_{p}$.

\subsection{Root Clashing}

Let $r_{1}, \ldots, r_{t}$ be the roots of $\Lambda_{1}(z)$ which is the output of the Berlekamp/Massey algorithm on the sequence of the outputs from the black box on the first set of evaluation points $\alpha_{1}, \ldots, \alpha_{n}$. Suppose at the $k$ 'th step, we want to compute the degrees of all the monomials in the variable $x_{k}$. As mentioned in the Example 1, the first step is to compute $\Lambda_{k+1}$. Then if $\operatorname{deg}_{x_{k}}\left(M_{i}\right)=e_{i k}$ we have $\bar{r}_{i}=r_{i} \times\left(\frac{\alpha_{n+1}}{\alpha_{k}}\right)^{e_{i k}}$ is a root of $\Lambda_{k+1}$. If $r_{i} \times\left(\frac{\alpha_{n+1}}{\alpha_{k}}\right)^{e^{\prime}}, 0 \leq e^{\prime} \neq e_{i k} \leq d$ is also a root of $\Lambda_{k+1}$ then we may not be able to uniquely identify the correct degree of the $i$ 'th monomial in the $k$ 'th variable $x_{k}$. We will illustrate this with an example.

Example 2 Consider the polynomial given in Example 1. Suppose instead of choosing $\alpha_{4}=34$, we choose $\alpha_{4}=72$ which is another generator of $\mathbb{Z}_{p}^{*}$. Since $\alpha_{1}, \alpha_{2}$ and $\alpha_{3}$ are the same as before, $\Lambda_{1}$ does not change and hence the roots of $\Lambda_{1}$ are $r_{1}=1, r_{2}=7, r_{3}=41, r_{4}=61$ and $r_{5}=64$. In the next step we substitute $\alpha_{4}=72$ for $\alpha_{1}$ and compute $\Lambda_{2}=z^{5}+61 z^{4}+39 z^{3}+67 z^{2}+37 z+98$. We proceed to compute the degrees of the monomials in $x$ but we find that

$$
r_{4} \times\left(\frac{\alpha_{4}}{\alpha_{1}}\right)^{2}=15 \text { and } r_{4} \times\left(\frac{\alpha_{4}}{\alpha_{1}}\right)^{4}=7
$$

are both roots of $\Lambda_{2}$ and hence we can not decide the correct degree of the last monomial in $x$.

Theorem 2 If $\operatorname{deg} \Lambda_{1}(z)=\operatorname{deg} \Lambda_{k+1}(z)=t$ then the probability that we cannot uniquely compute the degrees of all $M_{i}\left(x_{1}, \ldots, x_{n}\right)$ in $x_{k}$ is at most $\frac{d^{2} t^{2}}{4 \phi(p-1)}$.

Proof. Let $S_{i}=\left\{r_{j} \times\left(\frac{\alpha_{n+1}}{\alpha_{k}}\right)^{i} \mid 1 \leq j \leq t\right\}$ for $0 \leq$ $i \leq d$. We assume that $r_{i} \neq r_{j}$ for all $1 \leq i \neq j \leq t$. We will not be able to uniquely identify the degree of the $j$ 'th monomial in $x_{k}$ if there exists $\bar{d}$ such that $r_{j} \times\left(\frac{\alpha_{n+1}}{\alpha_{k}}\right)^{\bar{d}}=\bar{r}_{i}$ is a root of $\Lambda_{k+1}(z)$ and $0 \leq d \neq e_{j k} \leq d$ where $e_{j k}$ is $\operatorname{deg}_{x_{k}}\left(M_{j}\right)$. But we have $\bar{r}_{i}=r_{i} \times\left(\frac{\alpha_{n+1}}{\alpha_{k}}\right)^{e_{i k}}$ thus $r_{j} \times$ $\left(\frac{\alpha_{n+1}}{\alpha_{k}}\right)^{\bar{d}}=r_{i} \times\left(\frac{\alpha_{n+1}}{\alpha_{k}}\right)^{e_{i k}}$. Without loss of generality, assume $\tilde{d}=\bar{d}-e_{i k}>0$. We have $r_{i}=r_{j} \times\left(\frac{\alpha_{n+1}}{\alpha_{k}}\right)^{\tilde{d}}$ and hence 
$r_{i} \in S_{\tilde{d}} \Rightarrow S_{0} \cap S_{\tilde{d}} \neq \emptyset$. Hence we will not be able to compute the degrees in $x_{k}$ if $S_{0} \cap S_{i} \neq \emptyset$ for some $1 \leq i \leq d$. Let

$$
g(x)=\prod_{1 \leq l \neq j \leq t}\left(r_{j} x^{i}-r_{l} \alpha_{k}^{i}\right) .
$$

We have $r_{l}=r_{j} \times\left(\frac{\alpha_{n+1}}{\alpha_{k}}\right)^{i} \in S_{0} \cap S_{i}$ iff $g\left(\alpha_{n+1}\right)=0$. Using the Schwartz-Zippel lemma, the probability that $g\left(\alpha_{n+1}\right)=$ 0 is at most $\frac{\operatorname{deg} g}{\phi(p-1)}=\frac{\left(\begin{array}{c}t \\ 2\end{array}\right) i}{\phi(p-1)}<\frac{i t^{2}}{2 \phi(p-1)}$. If we sum this quantity for all $1 \leq i \leq d$ we obtain that the overall probability is at most $\frac{d^{2} t^{2}}{4 \phi(p-1)}$.

Using Theorem 2, the probability that we will not be able to uniquely identify the degrees of the monomials in all the variables is at most $\frac{n d^{2} t^{2}}{4 \phi(p-1)}$, i.e. for $p$, s.t. $\phi(p-1)>\frac{n d^{2} t^{2}}{2}$ with probability at least half, the algorithm succeeds without dealing with any problem. We will now discuss our solution to this problem. Note that we assume the images of the monomials are distinct, i.e. $\forall 1 \leq i \neq j \leq t, m_{i, k} \neq m_{j, k}$. Suppose we have computed $\Lambda_{k+1}$ and we want to compute the degrees of the monomials in $x_{k}$ and let $R_{1}=\left\{r_{1}, \ldots, r_{t}\right\}$ be the set of all the roots of $\Lambda_{1}$ and $R_{k}=\left\{\bar{r}_{1}, \ldots, \bar{r}_{t}\right\}$ be the set of all the distinct roots of $\Lambda_{k+1}$. Let

$$
D_{j}=\left\{(i, r) \mid 0 \leq i \leq d, r=r_{j} \times\left(\frac{\alpha_{n+1}}{\alpha_{1}}\right)^{i} \in R_{k}\right\} .
$$

$D_{j}$ contains the set of all possible degrees of the $j^{\prime}$ th monomial $M_{j}$ in the $k^{\prime}$ th variable $x_{k}$. We know that $\left(e_{j k}, \bar{r}_{j}\right) \in D_{j}$ and hence $\left|D_{j}\right| \geq 1$. If $\left|D_{j}\right|=1$ for all $1 \leq j \leq t$, then the degrees are unique and this step of the algorithm is complete. Let $G_{k}$ be a balanced bipartite graph defined as follows. $G_{k}$ has two independent sets of nodes $U$ and $V$ each of size $t$. Nodes in $U$ and $V$ represent elements in $R_{1}$ and $R_{k}$ respectively, i.e. $u_{i} \in U$ and $v_{j} \in V$ are labeled with $r_{i}$ and $\bar{r}_{j}$. We connect $u_{i} \in U$ to $v_{j} \in V$ with an edge of weight (degree) $d_{i j}$ if and only if $\left(d_{i j}, \bar{r}_{j}\right) \in D_{i}$.

Lemma 1 We can uniquely identify the degrees of all the monomials in $x_{k}$, if and only if the bipartite graph $G_{k}$ has a unique perfect matching.

The proof of this lemma is immediate by looking at the structure of the graph $G_{k}$. We illustrate with an example.

Example 3 Let $f$ be the polynomial given in Example 1 and suppose for some evaluation points $\alpha_{1}, \ldots, \alpha_{4}$ we obtain the graph $G_{1}$ as shown in Figure 1. This graph has a perfect matching, i.e. the set of edges $\left\{\left(r_{i}, \bar{r}_{i}\right) \mid 1 \leq i \leq 5\right\}$. If there was an edge connecting $r_{1}$ to $\bar{r}_{2}$ then the new graph would no longer have a unique perfect matching and we would fail to uniquely compute the degrees of monomials in $x$.

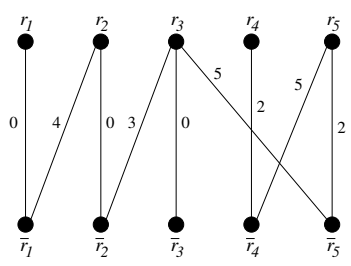

Figure 1: The bipartite graph $G_{1}$
We now give a solution for the case where $G_{k}$ does not have a unique perfect matching for some $1 \leq k \leq n$. The solution involves $2 t$ more probes to the black box. Suppose we choose a random element $\alpha_{n+2} \in \mathbb{Z}_{p}$ such that $\gamma=\frac{\alpha_{n+2}}{\alpha_{n+1}}$ is a generator of $\mathbb{Z}_{p}^{*}$ (or is of order greater than $d$ ). Let $\beta_{i}=\left(\alpha_{1}^{i}, \ldots, \alpha_{k-1}^{i}, \alpha_{n+2}^{i}, \alpha_{k+1}^{i}, \ldots, \alpha_{n}^{i}\right)$ and let $v_{i}$ be the output of the black box on input $\beta_{i}(0 \leq i \leq 2 t-1)$. On input $V=\left(\beta_{0}, \ldots, \beta_{2 t-1}\right)$, the Berlekamp/Massey algorithm computes a linear generator $\Lambda_{k+1}^{\prime}(z)$ for $V$. Let $\left\{\tilde{r}_{1}, \ldots, \tilde{r}_{t}\right\}$ be the set of distinct roots of $\Lambda_{k+1}^{\prime}$. Let $G_{k}^{\prime}$ be the balanced bipartite graph, obtained from $\Lambda_{1}$ and $\Lambda_{k+1}^{\prime}$.

Definition 1. We define $\bar{G}_{k}$, the intersection of $G_{k}^{\prime}$ and $G_{k}$, as follows. $\bar{G}_{k}$ has the same nodes as $G_{k}^{\prime}$ and there is an edge between $r_{i}$ and $\tilde{r}_{j}$ with weight (degree) $d_{i j}$ if and only if $r_{i}$ is connected to $\bar{r}_{j}$ in $G_{k}$ and to $\tilde{r}_{j}$ in $G_{k}^{\prime}$, both with the same degree $d_{i j}$.

Lemma 2 Let $e_{i j}=\operatorname{deg}_{x_{j}}\left(M_{i}\right)$. The two nodes $r_{i}$ and $\tilde{r}_{i}$ are connected in $\bar{G}_{k}$ with degree $e_{i j}$.

We take advantage of the following theorem which implies we need at most one extra set of probes.

Theorem 3 Let $\bar{G}_{k}=G_{k} \cap G_{k}^{\prime}$. $\bar{G}_{k}$ has a unique perfect matching.

Proof. Let $U$ and $V$ be the set of independent nodes in $\bar{G}_{k}$ such that $u_{i} \in U$ and $v_{j} \in V$ are labeled with $r_{i}$ and $\tilde{r}_{j}$ respectively where $\tilde{r}_{j}$ is a root of $\Lambda_{k+1}^{\prime}$. We will prove that each node in $V$ has degree exactly 1 and hence there is a unique perfect matching. The proof is by contradiction. Suppose the degree of $v_{j} \in V$ is at least 2. With out loss of generality assume that $r_{1}$ and $r_{2}$ are both connected to $\tilde{r}_{j}$ with degrees $d_{1 j}$ and $d_{2 j}$ respectively (See Figure 2).

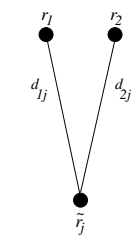

Figure 2: Node $\tilde{r}_{j}$ of graph $\bar{G}_{k}$

Using Definition 1 we have

$$
\begin{gathered}
\bar{r}_{j}=r_{1} \times\left(\frac{\alpha_{n+1}}{\alpha_{k}}\right)^{d_{1 j}}=r_{2} \times\left(\frac{\alpha_{n+1}}{\alpha_{k}}\right)^{d_{2 j}} \text { and } \\
\tilde{r}_{j}=r_{1} \times\left(\frac{\alpha_{n+2}}{\alpha_{k}}\right)^{d_{1 j}}=r_{2} \times\left(\frac{\alpha_{n+2}}{\alpha_{k}}\right)^{d_{2 j}} .
\end{gathered}
$$

Dividing the two sides of these equations results in

$$
\left(\frac{\alpha_{n+2}}{\alpha_{n+1}}\right)^{d_{1 j}}=\left(\frac{\alpha_{n+2}}{\alpha_{n+1}}\right)^{d_{2 j}} .
$$

Since we chose $\alpha_{n+2}$ such that $\frac{\alpha_{n+2}}{\alpha_{n+1}}$ has a sufficiently large order (greater than the degree bound $d$ ) we have $d_{1 j}=d_{2 j} \Rightarrow$ $r_{1}=r_{2}$. But this is a contradiction because both $r_{1}$ and $r_{2}$ are roots of $\Lambda_{1}$ which we assumed are distinct.

Lemma 2 and Theorem 3 prove that the intersection of $G_{k}$ and $G_{k}^{\prime}$ will give us the correct degrees of all the monomials in the $k$ 'th variable $x_{k}$. We will illustrate with an example. 
Example 4 Let $f=-10 y^{3}-7 x^{2} y z-40 y z^{5}+42 y^{3} z^{5}-$ $50 x^{7} z^{2}+23 x^{5} z^{4}+75 x^{7} y z^{2}-92 x^{6} y^{3} z+6 x^{3} y^{5} z^{2}+74 x y z^{8}+4$ and $p=101$. We choose the first set of evaluation points to be $\alpha_{1}=66, \alpha_{2}=11, \alpha_{3}=48$ and $\alpha_{4}=50$. For the first variable $x$ we will obtain the bipartite graph $G_{1}$ shown in Figure 3.

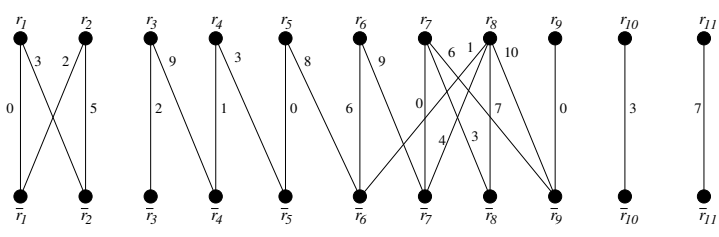

Figure 3: The bipartite graph $G_{1}$

This graph does not have a unique perfect matching, so we proceed to choose a new evaluation point $\alpha_{5}=89$. This time we will get the bipartite graph $G_{1}^{\prime}$ shown in Figure 4.

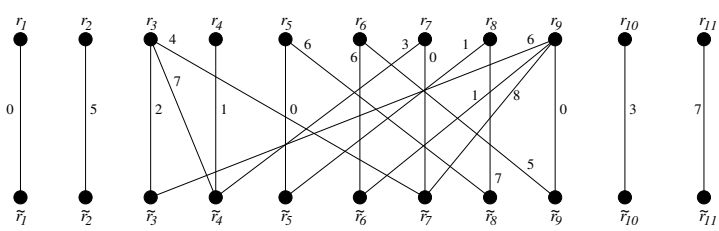

Figure 4: The bipartite graph $G_{1}^{\prime}$

Again $G_{1}^{\prime}$ does not have a unique perfect matching. We compute the intersection of $G_{1}$ and $G_{1}^{\prime}: \bar{G}_{1}=G_{1} \cap G_{1}^{\prime} . \bar{G}_{1}$ is shown in Figure 5.

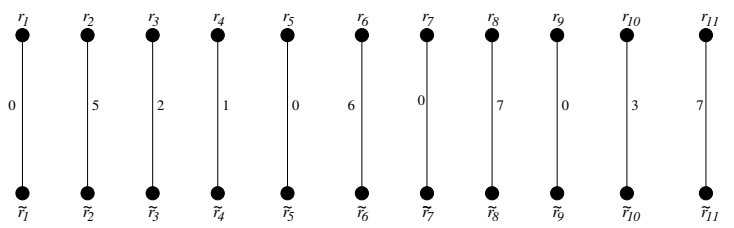

Figure 5: The bipartite graph $\bar{G}_{1}$

As stated by Theorem 3, $\bar{G}_{1}$ has a unique perfect matching and the degree of every monomial in $x$ is correctly computed.

In this section we proved that if the prime $p$ is sufficiently large $\left(\phi(p-1)\right.$ must be approximately $d t^{2}$ for us to be able to get distinct images of monomials with reasonable probability), we will be able to compute the degrees of all the $t$ monomials in each variable $x_{k}$ using up to $4 t$ evaluation points. If the graph $G_{k}$ has a unique perfect matching, we will be able to compute the degrees in $x_{k}$ with only $2 t$ probes to the black box.

We conclude this section with the following lemma which we will later use in Section 4.

Lemma 3 Let $G_{k}$ be the bipartite graph for the $k$ 'th variable. Let $u_{i_{1}} \rightarrow v_{j_{1}} \rightarrow u_{i_{2}} \rightarrow v_{j_{2}} \rightarrow \cdots \rightarrow v_{j_{s}} \rightarrow u_{i_{1}}$ be a cycle in $G_{k}$ where $u_{l} \in U$ is labeled with $r_{l}$ (a root of $\Lambda_{1}$ ) and $v_{m} \in V$ is labeled with $\bar{r}_{m}$ (a root of $\Lambda_{k+1}$ ). Let $d_{l m}$ be the weight (degree) of the edge between $u_{l}$ and $v_{m}$. We have $\sum_{m=1}^{s} d_{i_{m} j_{m}}-\sum_{m=1}^{s} d_{i_{m+1} j_{m}}=0$.
Proof. It is easy to show that $r_{i_{1}}=\left(\frac{\alpha_{n+1}}{\alpha_{k}}\right)^{\bar{d}} r_{i_{s}}$ where $\bar{d}=d_{i_{1} j_{1}}-d_{i_{2} j_{1}}+d_{i_{2} j_{2}}-d_{i_{3} j_{2}}+\cdots+d_{i_{s-1} j_{s-1}}-d_{i_{s} j_{s-1}}$. Also both $u_{i_{1}}$ and $u_{i_{s}}$ are connected to $v_{j_{s}}$ in $G_{k}$ hence we have $r_{i_{1}}=\left(\frac{\alpha_{n+1}}{\alpha_{k}}\right)^{d_{i_{1} j_{s}}} \bar{r}_{i_{s}}$ and $r_{i_{s}}=\left(\frac{\alpha_{n+1}}{\alpha_{k}}\right)^{d_{i_{s} j_{s}}} \bar{r}_{i_{s}}$. These three equations yield to $r_{i_{1}}=\left(\frac{\alpha_{n+1}}{\alpha_{k}}\right)^{\tilde{d}} r_{i_{1}}$ where $\tilde{d}=d_{i_{1} j_{1}}-$ $d_{i_{2} j_{1}}+d_{i_{2} j_{2}}-d_{i_{3} j_{2}}+\cdots+d_{i_{s-1} j_{s-1}}-d_{i_{s} j_{s-1}}+d_{i_{s} j_{s}}-d_{i_{1} j_{s}}$. But if $\frac{\alpha_{n+1}}{\alpha_{k}}$ is of sufficiently high order, $\tilde{d}$ must be zero thus $\sum_{m=1}^{s} d_{i_{m} j_{m}}-\sum_{m=1}^{s} d_{i_{m+1} j_{m}}=0$.

Example 5 In $G_{1}^{\prime}$ shown in Figure 4 , there is a cycle $r_{3} \rightarrow$ $\tilde{r}_{4} \rightarrow r_{7} \rightarrow \tilde{r}_{7} \rightarrow r_{3}$. The weights (degrees) of the edges in this cycle are as $7,3,0$ and 4 . We have $7-3+0-4=0$.

\section{THE ALGORITHM}

\section{Algorithm: Interpolation}

Input: A black box $\mathbf{B}: \mathbb{Z}_{p}^{n} \rightarrow \mathbb{Z}_{p}$ that on input $\alpha_{1}, \ldots, \alpha_{n} \in \mathbb{Z}_{p}^{n}$ outputs $f\left(\alpha_{1}, \ldots, \alpha_{n}\right)$ where $f \in \mathbb{Z}_{p}\left[x_{1}, \ldots, x_{n}\right]$.

Input: A degree bound $d \geq \operatorname{deg}(f)$.

Input: A bound $T \geq t$ on the number of terms in $f$.

Output: The polynomial $f$ or FAIL.

1: Choose $n+1$ generators $\alpha_{1} \neq \ldots \neq \alpha_{n+1}$ of $\mathbb{Z}_{p}^{*}$ randomly.

2: repeat choose $\gamma$ to be a random generator of $\mathbb{Z}_{p}^{*}$ and let $\alpha_{n+2}=\alpha_{n+1} \times \gamma$ until $\alpha_{n+2} \notin\left\{\alpha_{1}, \ldots, \alpha_{n+1}\right\}$.

3: Let $\beta_{i}=\left(\alpha_{1}^{i}, \ldots, \alpha_{n}^{i}\right)$ for $0 \leq i \leq 2 T-1$.

4: for $k$ from 1 to $n+1$ in parallel do

5: $\quad$ Compute $\Lambda_{k}(z)$ :

6: $\quad$ Compute $v_{i}=\mathbf{B}\left(\beta_{i}\right)$ for $0 \leq i \leq 2 t-1$ using $\alpha_{n+1}^{i}$ instead of $\alpha_{k-1}^{i}$ when $k>1$.

7: Use the Berlekamp/Massey algorithm to compute a linear generator $\Lambda_{k} \in \mathbb{Z}_{p}[z]$ for the sequence $v_{0}, \ldots, v_{2 t+1}$.

8: end for

9: Set $t=\max \left(\operatorname{deg} \Lambda_{1}(z), \ldots \Lambda_{n+1}(z)\right)$. If the degree of the $\Lambda$ 's are not all equal to $t$ then repeat steps 1 through step 8 once. If this does not yield equal degree $\Lambda$ 's then ( $p$ is likely too small so) return FAIL.

10: Compute $\left\{r_{1}, \ldots, r_{t}\right\}$ the set of distinct roots of $\Lambda_{1}(z)$.

11: for $k$ from 1 to $n$ in parallel do

12: $\quad$ Determine $\operatorname{deg}_{x_{k}}\left(M_{i}\right)$ for $1 \leq i \leq t$ :

13: $\quad$ Construct the graph $G_{k}$ as described in Section 3 .

14: if $G_{k}$ has a unique perfect matching then

15: $\quad$ Set $e_{i k}=d_{i l}$ where $d_{i l}$ is the weight (degree) of the edge that matches the node $r_{i}$ to $\bar{r}_{l}$ in the perfect matching. 16: else

17: $\quad$ Construct the graph $G_{k}^{\prime}$ as described in Section 3

Note, this requires $2 t$ more probes to $\mathbf{B}$.

18: $\quad$ Find the intersection of $G_{k}$ and $G_{k}^{\prime}: \bar{G}_{k}=G_{k} \cap G_{k}^{\prime}$.

19: $\quad$ Set $e_{i k}=d_{i l}$ where $d_{i l}$ is the weight (degree) of the edge that matches the node $r_{i}$ to $\tilde{r}_{l}$ in the perfect matching of graph $\bar{G}_{k}$.

20: end if

21: end for

22: Let $S=\left\{a_{1} r_{1}^{i}+a_{2} r_{2}^{i}+\cdots+a_{t} r_{t}^{i}=v_{i} \mid 0 \leq i \leq 2 t^{\prime}-1\right\}$ Solve the linear system $S$ for $\left(a_{1}, \ldots, a_{t}\right) \in \mathbb{Z}_{p}^{t}$.

23: Let $g=\sum_{i=1}^{t} a_{i} M_{i}$ where $M_{i}=\prod_{j=1}^{n} x_{j}^{e_{i j}}$.

24: Pick non-zero $a_{1}, \ldots, a_{n}$ from $\mathbb{Z}_{p}$ at random.

If $\mathbf{B}\left(a_{1}, \ldots, a_{n}\right) \neq g\left(a_{1}, \ldots, a_{n}\right)$ then return FAIL.

25: return $g$.

Remark 1 The algorithm is probabilistic. If the degrees of the $\Lambda^{\prime} s$ are all equal to $t$ then the algorithm will compute $f$ with probability 1 . If the degrees of the $\Lambda^{\prime} s$ are all equal but less than $t$ then the algorithm cannot compute $f$; that is, $g \neq$ $f$. The check in step 24 detects incorrect $g$ with probability at least $1-d /(p-1)$ (the Schwartz-Zippel lemma). Thus by doing one additional probe to the black box, we verify the output $g$ with high probability. Kaltofen and Lee in [11] also use additional probes to verify the output this way. 
Remark 2 For simplicity, our presentation of the algorithm assumes the term bound $T$ is good. In applications where a good term bound is not available, one should first compute $\Lambda_{1}(z)$ using $T$, and then use $t=\operatorname{deg} \Lambda_{1}(z)$ when computing $\Lambda_{2}, \ldots, \Lambda_{n+1}$.

\subsection{Complexity Analysis}

We now discuss the sequential complexity of the algorithm assuming $t=T$. We need to consider the cost of probing the black box. Let $E(n, t, d)$ be the cost of one probe to the black box. If $G_{k}$ has a unique perfect matching for $1 \leq k \leq n$ then we can correctly compute the degrees using only $G_{k}$. In this case the total number of probes is $2(n+1) t$ in the first loop. In the worst case where $G_{k}$ does not have a unique perfect matching for all $1 \leq k \leq n$, we need to do additional $2 n t$ probes to the black box in the second loop to construct all $G_{k}^{\prime}$ graphs. In this case the total number of probes to the black box is $2(n+1) t+2 n t=2(2 n+1) t$. Hence the total cost of probes to the black box is $O(n t E(n, t, d))$.

The $n+1$ calls to the Berlekamp/Massey algorithm in the first loop (as presented in [10]) cost $O\left(t^{2}\right)$ time each. The Vandermonde system of equations at Step 22 can be solved in $O\left(t^{2}\right)$ using the technique given in [18]. Note that as mentioned in [18], when inverting a $t \times t$ Vandemonde matrix defined by $k_{1}, \ldots, k_{t}$, one of the most expensive parts of this technique is to compute the master polynomial $M(z)=$ $\prod_{i=1}^{t}\left(z-k_{i}\right)$. However, in our algorithm we can use the fact that $M(z)=\prod_{i=1}^{t}\left(z-r_{i}\right)=\Lambda_{1}(z)$.

To compute the roots of $\Lambda_{1}(z)$ at Step 10 of the algorithm, we use Rabin's Las Vegas algorithm [15]. If $f \in \mathbb{Z}_{p}[z]$ is a product of linear factors, Rabin's algorithm tries to split it into two factors of lower degree by computing the $\operatorname{gcd}\left((z-\beta)^{(p-1) / 2}-1, \Lambda_{1}(z)\right)$ for randomly chosen $\beta \in \mathbb{Z}_{p}$. Since $\operatorname{deg}_{z}\left(\Lambda_{1}\right)=t$, the cost of finding the $t$ roots of $\Lambda_{1}(z)$, assuming classical algorithms for polynomial arithmetic in $\mathbb{Z}_{p}[z]$ are used, is $O\left(t^{2} \log p\right)$. See Algorithm 14.15 of [3].

We can compute the information needed to construct the bipartite graph $G_{k}$ in $O\left(d t^{2}\right)$ time. This involves evaluating $\Lambda_{k+1}(z)$ at $d$ points for each monomial and testing if it is zero or not. Also computing the intersection of $G_{k}$ and $G_{k}^{\prime}$ can be done in $O(t d \log d)$ time. This is because we know that each node in the intersection is of degree one (See proof of Theorem 3). Thus the overall time complexity is

$$
O\left(t^{2}(\log (p)+n d)+n t E(n, t, d)\right) .
$$

Remark 3 The algorithm, as presented, corresponds to our parallel implementation in Cilk. Further parallelism in the algorithm could be exploited. For example, one could compute all probes to the black box $\mathbf{B}$ in step 6 and step 17 in parallel. When determining the degree of the monomials in step 13 and 17, one can parallelize the evaluations of $\Lambda_{k+1}(z)$. The most expensive sequential component is the computation of the roots of $\Lambda_{1}(z)$ in step 10 which has complexity $O\left(t^{2} \log p\right)$. With asymptotically fast arithmetic this is $\tilde{O}(t \log p)$.

\subsection{Optimizations}

Let $D=\operatorname{deg}(f)$. If the prime $p$ is large enough, i.e. $p>\frac{n D^{2} t^{2}}{4 \epsilon}$ then with probability $1-\epsilon$ the degree of every monomial in $x_{k}$ can correctly be computed using only $G_{k}$ and without needing any extra probes to the black box. In fact in this case, with high probability, every $r_{i}$ will be matched with exactly only one $\bar{r}_{j}$ and hence every node in $G_{k}$ would have degree one (e.g. see Figure 5). But if $d \gg D$, i.e. the degree bound $d$ is not tight, the probability that we could identify the degrees uniquely drops significantly even though $p$ is large enough. This is because the probability that root clashing (see Section 3 ) happens, linearly depends on $d$. In this case, with probability $1-\epsilon$, the degree of $M_{i}$ in $x_{k}$ would be $\min \left\{d_{i j} \mid\left(d_{i j}, r_{i}\right) \in G_{k}\right\}$, i.e. the edge connected to $r_{i}$ in $G_{k}$ with minimum weight (degree) is our desired edge in the graph which will show up in the perfect matching. We apply the following theorem.

Theorem 4 Let $H_{k}$ be a graph obtained by eliminating all edges connected to $r_{i}$ in $G_{k}$ except the one with minimum weight (degree) for all $1 \leq i \leq t$. If the degree of every node in $H_{k}$ is exactly one, then $e_{i k}$ is equal to the weight of the edge connected to $r_{i}$ in $H_{k}$.

This theorem can be proved using Lemma 3 and the fact that there can not be any cycle in the graph $H_{k}$. We will give an example.

Example 6 Let $f=25 y^{2} z+90 y z^{2}+93 x^{2} y^{2} z+60 y^{4} z+42 z^{5}$. Here $t=5, n=3, d_{\max }=5$ and $p=101$. We choose the following evaluation points $\alpha_{1}=85, \alpha_{2}=96, \alpha_{3}=58$ and $\alpha_{4}=99$. Suppose we want to construct $G_{2}$ in order to compute the degrees of the monomials in $y$. Suppose our degree bound is $d=40$ which is not tight. The graph $G_{2}$ and $\mathrm{H}_{2}$ are shown in Figures 6 and 7 respectively.

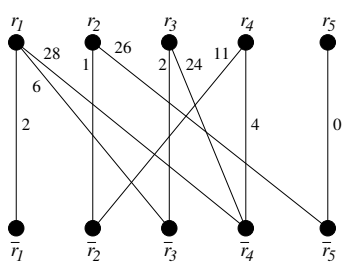

Figure 6: The bipartite graph $G_{2}$

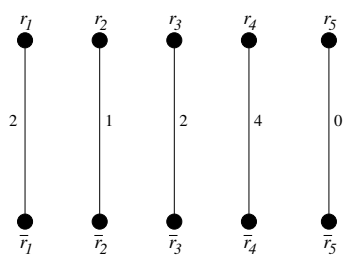

Figure 7: The bipartite graph $H_{2}$

The graph $\mathrm{H}_{2}$ has the correct degrees of the monomials in variable $y$.

Theorem 4 suggests the following optimization. In the construction of the bipartite graph $G_{k}$, connect $r_{i}$ to $\bar{r}_{j}$ with degree $d_{i j}$ only if there is no $\bar{d}<d_{i j}$ such that $r_{i} \times\left(\frac{\alpha_{n+1}}{\alpha_{k}}\right)^{\bar{d}}$ is a root of $\Lambda_{k+1}$, i.e. the degree of the node $r_{i}$ in $U$ is always one for all $1 \leq i \leq n$. If there is a perfect matching in this graph, this perfect matching is unique because this implies that the degree of each node $\bar{r}_{j}$ in $V$ is also one (e.g. see Figure 7). If not, go back to and complete the graph $G_{k}$. This optimization makes our algorithm sensitive to the actual degree of $f\left(x_{1}, \ldots, x_{n}\right)$ in each variable. 
The second optimization is to compute the degree of each monomial $M_{i}=x_{1}^{e_{i 1}} x_{2}^{e_{i 2}} \ldots x_{n}^{e_{i n}}$ in the last variable $x_{n}$ without doing any more probes to the black box. Suppose we have computed the degree of $M_{i}$ in $x_{k}$ for $1 \leq k<n$. We know that $M_{i}\left(\alpha_{1}, \ldots, \alpha_{n}\right)$ is equal to $r_{i}$, a root of $\Lambda_{1}$. Hence $r_{i}=\alpha_{1}^{e_{i 1}} \cdot \alpha_{2}^{e_{i 2}} \cdots \cdots \alpha_{n}^{e_{i n}}$. Since we know the degrees $e_{i j}$ for $1 \leq j<n$ we can determine $e_{i n}$ by division by $\alpha_{n}$. This reduces the total number of probes from $4(n+1) t$ to $4 n t$.

\section{BENCHMARKS}

Here, we compare the performance of our new algorithm, Zippel's algorithm and the racing algorithm of Kaltofen and Lee from [11]. We have implemented Zippel's algorithm and our new algorithm in C. We have also implemented an interface to call the interpolation routines from Maple. The racing algorithm is implemented in Maple in the ProtoBox package by Lee [11]. Since this algorithm is not coded in C, we only report (see columns labelled PBox) the number of probes it makes to the black box.

We give benchmarks comparing the performances on five problem sets. The polynomials in the first four benchmarks were generated at random. The fifth set of polynomials are taken from [11]. We count the number of probes to the black box and measure the total CPU time (for our new algorithm and Zippel's algorithm only). All the timings given in this section are in CPU seconds and were obtained using Maple 13 on a 64 bit Intel Core i7 920 @ 2.66GHz, running Linux. This is a 4 core machine. For our algorithm, we report the real time for 1 core and (in parentheses) 4 cores.

The black box in our benchmarks computes a multivariate polynomial with coefficients in $\mathbb{Z}_{p}$ where $p=3037000453$ is a 31.5 bit prime. In all benchmarks, the black box simply evaluates the polynomial at the given evaluation point. To evaluate efficiently we compute and cache the values of $x_{i}^{j}$ $\bmod p$ in a loop in $O(n d)$. Then we evaluate the $t$ terms in $O(n t)$. Hence the cost of one black box probe is $O(n d+n t))$ arithmetic operations in $\mathbb{Z}_{p}$.

\section{Benchmark \#1}

This set of problems consists of 13 multivariate polynomials in $n=3$ variables. The $i$ 'th polynomial $(1 \leq i \leq 13)$ is generated at random using the following Maple command:

$>\operatorname{randpoly}\left([\mathrm{x} 1, \mathrm{x} 2, \mathrm{x} 3]\right.$, terms $=2^{\wedge} i$, degree $\left.=30\right) \bmod \mathrm{p}$;

The $i$ 'th polynomial will have about $2^{i}$ non-zero terms. Here $D=30$ is the total degree hence the maximum number of terms in each polynomial is $t_{\max }=\left(\begin{array}{c}n+D \\ D\end{array}\right)=5456$. We run both the Zippel's algorithm and our new algorithm with degree bound $d=30$. The timings and the number of probes are given in Table 1. In this table "DNF" means that the algorithm did not finish after 12 hours.

As $i$ increases, the polynomial $f$ becomes denser. For $i>6$, $f$ has more than $\sqrt{t_{\max }}$ non-zero terms. This is indicated by a horizontal line in Table 1 and also in subsequent benchmarks. The line approximately separates sparse inputs from dense inputs. The last polynomial $(i=13)$ is $99.5 \%$ dense.

The data in Table 1 shows that for sparse polynomials $1 \leq i \leq 6$, our new algorithm does a lot fewer probes to the black box compared to Zippel's algorithm. It also does fewer probes than the racing algorithm (PBox). However, as the polynomials get denser, Zippel's algorithm has a better performance. For a completely dense polynomial with $t$ non-

\begin{tabular}{|c|c|c|c|c|c|c|}
\hline$i$ & $t$ & \multicolumn{2}{|c|}{ New Algorithm } & \multicolumn{2}{|c|}{ Zippel } & PBox \\
\cline { 3 - 7 } & & Time & Probes & Time & Probes & Probes \\
\hline 1 & 2 & $0.00(0.00)$ & 12 & 0.00 & 217 & 20 \\
2 & 4 & $0.00(0.00)$ & 24 & 0.00 & 341 & 39 \\
3 & 8 & $0.00(0.00)$ & 48 & 0.00 & 558 & 79 \\
4 & 16 & $0.00(0.00)$ & 96 & 0.01 & 868 & 156 \\
5 & 32 & $0.00(0.00)$ & 192 & 0.01 & 1519 & 282 \\
6 & 64 & $0.01(0.01)$ & 384 & 0.03 & 2573 & 517 \\
\hline 7 & 128 & $0.03(0.02)$ & 768 & 0.08 & 4402 & 962 \\
8 & 253 & $0.11(0.06)$ & 1518 & 0.21 & 6417 & 1737 \\
9 & 512 & $0.44(0.24)$ & 3072 & 0.55 & 9734 & 3119 \\
10 & 1015 & $1.66(0.88)$ & 6090 & 1.16 & 12400 & 5627 \\
11 & 2041 & $6.50(3.44)$ & 12246 & 2.43 & 15128 & DNF \\
12 & 4081 & $25.3(13.4)$ & 24486 & 4.56 & 16182 & DNF \\
13 & 5430 & $44.3(23.3)$ & 32580 & 5.93 & 16430 & DNF \\
\hline
\end{tabular}

Table 1: benchmark \#1: $n=\mathbf{3}$ and $D=\mathbf{3 0}$

zero terms, Zippel's algorithm only does $O(t)$ probes to the black box while the new algorithm does $O(n t)$ probes.

To show how effective the first optimization described in Section 4.2 is, we run both our algorithm and Zippel's algorithm on the same set of polynomials but with a bad degree bound $d=100$. The timings and the number of probes are given in Table 2. One can see that our algorithm is unaffected by the bad degree bound; the number of probes and CPU timings are the same.

\begin{tabular}{|c|c|c|c|c|c|}
\hline$i$ & \multirow{2}{*}{$t$} & \multicolumn{2}{|c|}{ New Algorithm } & \multicolumn{2}{c|}{ Zippel's Algorithm } \\
\cline { 3 - 6 } & & Time & Probes & Time & Probes \\
\hline 1 & 2 & $0.00(0.00)$ & 12 & 0.01 & 707 \\
2 & 4 & $0.00(0.00)$ & 24 & 0.01 & 1111 \\
3 & 8 & $0.00(0.00)$ & 48 & 0.02 & 1818 \\
4 & 16 & $0.00(0.00)$ & 96 & 0.03 & 2828 \\
5 & 32 & $0.00(0.00)$ & 192 & 0.07 & 4949 \\
6 & 64 & $0.01(0.01)$ & 384 & 0.14 & 8383 \\
\hline 7 & 128 & $0.04(0.02)$ & 768 & 0.36 & 14342 \\
8 & 253 & $0.12(0.07)$ & 1518 & 0.79 & 20907 \\
9 & 512 & $0.45(0.24)$ & 3072 & 1.97 & 31714 \\
10 & 1015 & $1.67(0.89)$ & 6090 & 3.97 & 40400 \\
11 & 2041 & $6.50(3.45)$ & 12246 & 8.18 & 49288 \\
12 & 4081 & $25.3(13.4)$ & 24486 & 15.16 & 52722 \\
13 & 5430 & $44.1(23.4)$ & 32580 & 19.62 & 53530 \\
\hline
\end{tabular}

Table 2: benchmark \#1: bad degree bound $d=100$

\section{Benchmark \#2}

In this set of benchmarks the $i$ 'th polynomial is in $n=3$ variables and is generated at random in Maple using

$>\operatorname{randpoly}\left([\mathrm{x} 1, \mathrm{x} 2, \mathrm{x} 3]\right.$, terms $=2^{\wedge} \mathrm{i}$, degree $\left.=100\right) \bmod \mathrm{p}$;

This set of polynomials differs from the first benchmark in that the total degree of each polynomial is set to be 100 in the second set. We run both the Zippel's algorithm and our new algorithm with degree bound $d=100$. The timings and the number of probes are given in Table 3. Comparing this table to the data in Table 1 shows that the number of probes to the black box in our new algorithm does not depend on the degree of the target polynomial. 


\begin{tabular}{|c|c|c|c|c|c|c|}
\hline$i$ & $t$ & \multicolumn{2}{|c|}{ New Algorithm } & \multicolumn{2}{c|}{ Zippel } & PBox \\
\cline { 3 - 7 } & & Time & Probes & Time & Probes & Probes \\
\hline 1 & 2 & $0.00(0.00)$ & 12 & 0.01 & 707 & 19 \\
2 & 4 & $0.00(0.00)$ & 24 & 0.01 & 1111 & 45 \\
3 & 8 & $0.00(0.00)$ & 48 & 0.02 & 1919 & 89 \\
4 & 16 & $0.00(0.00)$ & 96 & 0.04 & 3434 & 167 \\
5 & 31 & $0.00(0.00)$ & 186 & 0.08 & 6161 & 320 \\
6 & 64 & $0.02(0.01)$ & 384 & 0.19 & 10504 & 623 \\
7 & 127 & $0.05(0.02)$ & 762 & 0.49 & 18887 & 1149 \\
8 & 253 & $0.17(0.09)$ & 1518 & 1.38 & 32219 & 2137 \\
\hline 9 & 511 & $0.66(0.34)$ & 3066 & 4.36 & 56863 & 4103 \\
10 & 1017 & $2.54(1.31)$ & 6102 & 13.99 & 98677 & 7836 \\
11 & 2037 & $9.83(5.09)$ & 12222 & 43.23 & 166650 & DNF \\
12 & 4076 & $38.7(19.9)$ & 24456 & 121.68 & 262802 & DNF \\
13 & 8147 & $152 .(78.3)$ & 48882 & 282.83 & 359863 & DNF \\
\hline
\end{tabular}

Table 3: benchmark \#2: $n=3$ and $D=100$

\section{Benchmarks \#3 and \#4}

These sets of problems consist of 14 random multivariate polynomials in $n=6$ variables and $n=12$ variables all of total degree $D=30$. The $i$ 'th polynomial will have about $2^{i}$ non-zero terms. We run both the Zippel's algorithm and our new algorithm with degree bound $d=30$. The timings and the number of probes are given in Tables 4 and 5 .

\begin{tabular}{|c|c|c|c|c|c|c|}
\hline$i$ & $t$ & \multicolumn{2}{|c|}{ New Algorithm } & \multicolumn{2}{c|}{ Zippel } & PBox \\
\cline { 3 - 7 } & & Time & Probes & Time & Probes & Probes \\
\hline 1 & 2 & $0.00(0.00)$ & 24 & 0.01 & 496 & 37 \\
2 & 3 & $0.00(0.00)$ & 36 & 0.01 & 651 & 59 \\
3 & 8 & $0.00(0.00)$ & 96 & 0.01 & 1364 & 140 \\
4 & 16 & $0.00(0.00)$ & 192 & 0.02 & 2511 & 284 \\
5 & 31 & $0.00(0.00)$ & 372 & 0.05 & 4340 & 521 \\
6 & 64 & $0.02(0.01)$ & 768 & 0.15 & 8060 & 995 \\
7 & 127 & $0.06(0.03)$ & 1524 & 0.44 & 14601 & 1871 \\
8 & 255 & $0.21(0.09)$ & 3060 & 1.51 & 27652 & 3615 \\
9 & 511 & $0.81(0.35)$ & 6132 & 5.19 & 50530 & 6692 \\
10 & 1016 & $3.10(1.33)$ & 12192 & 17.94 & 90985 & 12591 \\
\hline 11 & 2037 & $12.2(5.21)$ & 24444 & 65.35 & 168299 & DNF \\
12 & 4083 & $48.1(20.5)$ & 48996 & 230.60 & 301320 & DNF \\
13 & 8151 & $189 .(80.1)$ & 97812 & 803.26 & 532549 & DNF \\
\hline
\end{tabular}

Table 4: benchmark \#3: $n=6$ and $D=30$

To assess the parallel implementation of our algorithm, Table 6 reports timings for benchmark \#4 for our algorithm running on 1, 2 and 4 cores showing the speedup we obtain using 2 and 4 cores. We report (in column roots) the time spent computing the roots in step 10 of $\Lambda_{1}(z)$ using our implementation of Rabin's algorithm which uses classical polynomial arithmetic, and (in column solve) the time solving the linear system for the coefficients in step 22 and (in column probes) the total time spent probing the black box. The data shows that computing the roots will become a bottleneck for our parallel implementation for more cores. Thus for 2 cores and 4 cores we report two timings. The first (in column time 1) is for our parallel algorithm as presented. For the second (faster) time (in column time 2) we have parallelized the second and subsequent steps of the root finding algorithm which yields a modest speedup. The data

\begin{tabular}{|c|c|c|c|c|c|c|}
\hline$i$ & $t$ & \multicolumn{2}{|c|}{ New Algorithm } & \multicolumn{2}{|c|}{ Zippel } & PBox \\
\cline { 3 - 7 } & & Time & Probes & Time & Probes & Probes \\
\hline 1 & 2 & $0.00(0.00)$ & 44 & 0.03 & 1736 & 67 \\
2 & 4 & $0.00(0.00)$ & 96 & 0.04 & 3038 & 121 \\
3 & 8 & $0.00(0.00)$ & 192 & 0.08 & 5053 & 250 \\
4 & 15 & $0.00(0.00)$ & 360 & 0.20 & 10230 & 470 \\
5 & 32 & $0.02(0.01)$ & 768 & 0.54 & 18879 & 962 \\
6 & 63 & $0.04(0.02)$ & 1512 & 1.79 & 36735 & 1856 \\
7 & 127 & $0.15(0.05)$ & 3048 & 6.10 & 69595 & 3647 \\
8 & 255 & $0.54(0.17)$ & 6120 & 22.17 & 134664 & 7055 \\
9 & 507 & $2.01(0.60)$ & 12168 & 83.44 & 259594 & 13440 \\
10 & 1019 & $7.87(2.33)$ & 24456 & 316.23 & 498945 & 26077 \\
11 & 2041 & $31.0(9.16)$ & 48984 & 1195.13 & 952351 & DNF \\
12 & 4074 & $122.3(35.9)$ & 97776 & 4575.83 & 1841795 & DNF \\
13 & 8139 & $484.6(141)$. & 195336 & $>10000$ & - & DNF \\
\hline
\end{tabular}

Table 5: benchmark \#4: $n=12$ and $D=30$

can be interpreted as follows. For $i=13$ the sequential time is $484.6 \mathrm{~s}$. Of this, $34.7 \mathrm{~s}$ was spent computing the roots of $\Lambda_{1}(z)$ and $5.02 \mathrm{~s}$ was spent solving for the coefficients. Thus the algorithm has a sequential component of $34.7+5.02=$ $39.7 \mathrm{~s}$ and so the maximum possible speedup on 4 cores is a factor of $484.6 /((484.6-39.7) / 4+39.7)=3.21$ compared with the observed speedup factor of 484.6/152.5 = 3.18. One way to remove this bottleneck would be to use a fast multiplication and division algorithm for $\mathbb{Z}_{p}[z]$.

\section{Benchmark \#5}

In this benchmark, we compare our new algorithm and the racing algorithm on seven target polynomials (below) from $\left[11\right.$, p. 393]. Note, $f_{6}$ is dense. The number of probes for each algorithm is reported in Table 7 .

$$
\begin{gathered}
f_{1}\left(x_{1}, \ldots, x_{9}\right)=x_{1}^{2} x_{3}^{3} x_{4} x_{6} x_{8} x_{9}^{2}+x_{1} x_{2} x_{3} x_{4}^{2} x_{5}^{2} x_{8} x_{9}+ \\
x_{2} x_{3} x_{4} x_{5}^{2} x_{8} x_{9}+x_{1} x_{3}^{3} x_{4}^{2} x_{5}^{2} x_{6}^{2} x_{7} x_{8}^{2}+x_{2} x_{3} x_{4} x_{5}^{2} x_{6} x_{7} x_{8}^{2} \\
f_{2}\left(x_{1}, \ldots, x_{10}\right)=x_{1} x_{2}^{2} x_{4}^{2} x_{8} x_{9}^{2} x_{10}^{2}+x_{2}^{2} x_{4} x_{5}^{2} x_{6} x_{7} x_{9} x_{10}^{2}+ \\
x_{1}^{2} x_{2} x_{3} x_{5}^{2} x_{7}^{2} x_{9}^{2}+x_{1} x_{3}^{2} x_{4}^{2} x_{7}^{2} x_{9}^{2}+x_{1}^{2} x_{3} x_{4} x_{7}^{2} x_{8}^{2} \\
f_{3}\left(x_{1}, \ldots, x_{9}\right)=9 x_{2}^{3} x_{3}^{3} x_{5}^{2} x_{6}^{2} x_{8}^{3} x_{9}^{3}+9 x_{1}^{3} x_{2}^{2} x_{3}^{3} x_{5}^{2} x_{7}^{2} x_{8}^{2} x_{9}^{3}+ \\
x_{1}^{4} x_{3}^{4} x_{4}^{2} x_{5}^{4} x_{6}^{4} x_{7} x_{8}^{5} x_{9}+10 x_{1}^{4} x_{2} x_{3}^{4} x_{4}^{4} x_{5}^{4} x_{7} x_{8}^{3} x_{9}+12 x_{2}^{3} x_{4}^{3} x_{6}^{3} x_{7}^{2} x_{8}^{3} \\
f_{4}\left(x_{1}, \ldots, x_{9}\right)=9 x_{1}^{2} x_{3} x_{4} x_{6}^{3} x_{7}^{2} x_{8} x_{10}^{4}+17 x_{1}^{3} x_{2} x_{5}^{2} x_{6}^{2} x_{7} x_{8}^{3} x_{9}^{4} x_{10}^{3}+ \\
3 x_{1}^{3} x_{2}^{2} x_{6}^{3} x_{10}^{2}+17 x_{2}^{2} x_{3}^{4} x_{4}^{2} x_{7}^{4} x_{8}^{3} x_{9} x_{10}^{3}+10 x_{1} x_{3} x_{5}^{2} x_{6}^{2} x_{7}^{4} x_{8}^{4} \\
f_{5}\left(x_{1}, \ldots, x_{50}\right)=\sum_{i=5}^{i=50} x_{i}^{50} \\
f_{6}\left(x_{1}, \ldots, x_{5}\right)=\sum_{i=1}^{i=5}\left(x_{1}+x_{2}+x_{3}+x_{4}+x_{5}\right)^{i} \\
f_{7}\left(x_{1}, x_{2}, x_{3}\right)=x_{1}^{20}+2 x_{2}+2 x_{2}^{2}+2 x_{2}^{3}+2 x_{2}^{4}+3 x_{3}^{20}
\end{gathered}
$$

\begin{tabular}{|c|ccc|c|c|}
\hline$i$ & $n$ & $d$ & $\# f_{i}$ & New Algorithm & ProtoBox \\
\hline 1 & 9 & 3 & 5 & 90 & 126 \\
2 & 10 & 2 & 5 & 100 & 124 \\
3 & 9 & 3 & 5 & 90 & 133 \\
4 & 9 & 4 & 5 & 100 & 133 \\
5 & 50 & 50 & 50 & 5000 & 251 \\
6 & 5 & 5 & 251 & 2510 & 881 \\
7 & 3 & 20 & 6 & 36 & 41 \\
\hline
\end{tabular}

Table 7: benchmark \#5. 


\begin{tabular}{|c|c|cccc|cccc|ccc|}
\hline \multicolumn{5}{|c|}{} & \multicolumn{5}{|c|}{ 1 core } & \multicolumn{4}{c|}{ 2 cores } & \multicolumn{3}{c|}{4 cores } \\
\hline$i$ & $t$ & time & roots & solve & probe & time 1 & time 2 & (speedup) & time 1 & time 2 & (speedup) \\
\hline 6 & 63 & 0.04 & 0.01 & 0.00 & 0.04 & 0.03 & 0.02 & & 0.02 & 0.02 & \\
7 & 127 & 0.15 & 0.02 & 0.00 & 0.15 & 0.08 & 0.08 & $(1.87 \mathrm{x})$ & 0.06 & 0.05 & $(3 \mathrm{x})$ \\
8 & 255 & 0.54 & 0.05 & 0.00 & 0.41 & 0.30 & 0.28 & $(1.93 \mathrm{x})$ & 0.18 & 0.17 & $(3.18 \mathrm{x})$ \\
9 & 507 & 2.02 & 0.18 & 0.02 & 1.48 & 1.11 & 1.06 & $(1.91 \mathrm{x})$ & 0.67 & 0.60 & $(3.37 \mathrm{x})$ \\
10 & 1019 & 7.94 & 0.65 & 0.08 & 5.76 & 4.35 & 4.17 & $(1.90 \mathrm{x})$ & 2.58 & 2.33 & $(3.41 \mathrm{x})$ \\
11 & 2041 & 31.3 & 2.47 & 0.32 & 22.7 & 17.1 & 16.3 & $(1.92 \mathrm{x})$ & 9.94 & 9.16 & $(3.42 \mathrm{x})$ \\
12 & 4074 & 122.3 & 9.24 & 1.26 & 90.0 & 67.1 & 64.7 & $(1.89 \mathrm{x})$ & 38.9 & 35.9 & $(3.41 \mathrm{x})$ \\
13 & 8139 & 484.6 & 34.7 & 5.02 & 357.3 & 264.9 & 255.8 & $(1.89 \mathrm{x})$ & 152.5 & 141.5 & $(3.42 \mathrm{x})$ \\
\hline
\end{tabular}

Table 6: Parallel speedup timing data for benchmark \#4 for the new algorithm.

\section{CONCLUSION}

Our sparse interpolation algorithm is a modification of the Ben-Or/Tiwari algorithm [1] for polynomials over finite fields. It does a factor of between $n$ and $2 n$ more probes where $n$ is the number of variables. Our benchmarks show that for sparse polynomials, it usually does fewer probes to the black box than Zippel's algorithm and the racing algorithm of Kaltofen and Lee. Unlike Zippel's algorithm and the racing algorithm, our algorithm does not interpolate each variable sequentially and thus can more easily be parallelized. Our parallel implementation using Cilk, which parallelized only the main loops, demonstrates a good speedup. The downside of our algorithm is that it is clearly worse than Zippel's algorithm and the racing algorithm for dense polynomials. This disadvantage is partly compensated for by the increased parallelism.

Although we presented our algorithm for interpolating over $\mathbb{Z}_{p}$, it also works over any finite field $G F(q)$. Furthermore, if $p$ (or $q$ ) is too small, one can work inside a suitable extension field. We conclude with some remarks about the choice of $p$ in applications where one may choose $p$.

Theorem 1 says that monomial collisions are likely when $\frac{d t^{2}}{2 \phi(p-1)}>\frac{1}{2}$, that is when $\phi(p-1)<d t^{2}$. In our benchmarks we used the 31.5 bit prime 3037000453 . This is the biggest prime that we can use when programming in $\mathrm{C}$ on a 64 bit machine using signed 64 bit machine integers. Using this prime, if $d=30$, monomial collisions will likely occur when $t>5,808$ which means 31.5 bit primes are too small for large applications.

It is not difficult to choose $p$ so that $p-1=2 q$ with $q$ also prime. The largest such 31.5 bit prime is 3037000427 . Solving $\phi(p-1)<d t^{2}$ for $t$ with $d=30$ using this prime gives $t>7,114$. This choice of prime also makes it easy to find generators. However, for $p-1=2 q$, since -1 is the only element of order 2 , any value from the interval $[2, p-2]$ will have order $q$ or $2 q$. If we use elements of order $q$ as well as generators in our algorithm, then the probability that two monomials collide is less than $d t^{2} /(2 p-6)$ (using $|S|=p-3$ in the proof of Theorem 1). Solving $p-3<d t^{2}$ for $t$ using $d=30$ and $p=3037000427$ yields $t>10,061$.

The 31.5 bit prime limitation is not a limitation of the hardware, but of the $\mathrm{C}$ programming language. On a 64 bit machine, one can use 63 bit primes if one programs multiplication in assembler. We are presently implementing this.

\section{ACKNOWLEDGMENTS}

We would like to thank Wen-shin Lee for making the source code of the ProtoBox Maple package available to us.

\section{REFERENCES}

[1] M. Ben-Or and P. Tiwari. A deterministic algorithm for sparse multivariate polynomial interpolation. In Proc. of the twentieth annual ACM symposium on Theory of computing, pages 301-309. ACM, 1988.

[2] Cilk 5.4.6 Reference Manual, http://supertech.csail.mit.edu/cilk/manual-5.4.6.pdf. Supercomputing Technologies Group, MIT Lab for Computer Science. http://supertech.lcs.mit.edu/cilk.

[3] J. von zur Gathen and J. Gerhard. Modern Computer Algebra. Cambridge University Press, 2003.

[4] M. Giesbrecht, G. Labahn and W. Lee. Symbolic-numeric sparse interpolation of multivariate polynomials. J. Symb. Comput., 44:943-959, 2009.

[5] D. Grigoriev, M. Karpinski, and M. Singer. Fast parallel algorithms for sparse multivariate polynomial interpolation over finite fields. SIAM J. Comput. 19:1059-1063, 1990.

[6] M. A. Huang and A. J. Rao. Interpolation of sparse multivariate polynomials over large finite fields with applications. Journal of Algorithms, 33:204-228, 1999.

[7] S. M. M. Javadi and M. Monagan. A sparse modular gcd algorithm for polynomials over algebraic function fields. In Proc. of ISSAC'O7, pages 187-194. ACM, 2007.

[8] E. Kaltofen and Y. N. Lakshman. Improved sparse multivariate polynomial interpolation algorithms. In Proc. of ISSAC '88, pages 467-474. Springer-Verlag, 1989.

[9] E. Kaltofen, Y. N. Lakshman, and J.-M. Wiley. Modular rational sparse multivariate polynomial interpolation. In Proc. of ISSAC' '90, pages 135-139. ACM, 1990.

[10] E. Kaltofen, W. Lee, and A. A. Lobo. Early termination in Ben-Or/Tiwari sparse interpolation and a hybrid of Zippel's algorithm. In Proc. of ISSAC '00, pages 192-201. ACM, 2000.

[11] E. Kaltofen and W. Lee . Early termination in sparse interpolation algorithms. J. Symb. Comput., 36(3-4):365-400, 2003.

[12] J. L. Massey. Shift-register synthesis and bch decoding. IEEE Trans. on Information Theory, 15:122-127, 1969

[13] M. Monagan J. de Kleine and A. Wittkopf. Algorithms for the non-monic case of the sparse modular gcd algorithm. In Proc. of ISSAC '05, pages 124-131. ACM, 2005.

[14] S. Pohlig and M. Hellman. An improved algorithm for computing logarithms over GF(p) and its cryptographic significance. IEEE Trans. on Information Theory, 24:106-110, 1978.

[15] Michael O. Rabin. Probabilistic algorithms in finite fields. SIAM J. Comput, 9:273-280, 1979.

[16] Jack Schwartz, Fast probabilistic algorithms for verification of polynomial identities. J. ACM, 27:701-717, 1980.

[17] Richard Zippel. Probabilistic algorithms for sparse polynomials. In Proc. of EUROSAM 'r9, pages 216-226. Springer-Verlag, 1979.

[18] Richard Zippel. Interpolating polynomials from their values. J. Symb. Comput., 9(3):375-403, 1990. 